\title{
Fat Embolism Syndrome - Diagnostic Dilemma During Covid-19 Pandemic
}

\author{
Nimisha Mahesh Parkar ${ }^{\text {, Frida Gomes }}{ }^{2}$, Prabhav Pramod Dessai ${ }^{3}$, Chandia Rose Mendes ${ }^{4}$, Priyanka Suresh Phadte ${ }^{5}$
}

1, 2, 4, 5 Department of Anaesthesiology, Goa Medical College, Bambolim, Goa, India.

${ }^{3}$ Department of Orthopaedics, Goa Medical College, Bambolim, Goa, India.

\section{INTRODUCTION}

Acute onset of breathlessness and type 1 respiratory failure in young patients in the orthopaedic ward with traumatic pelvic or long bone fractures often raise suspicion of thromboembolic event, classic fat embolism and fat embolism syndrome (FES). However, in the era of the prevailing pandemic, the clinical presentation may cause suspicion of Covid-19 infection, creating a dilemma for diagnostic purposes, causing inadvertent delays in definitive management.

Although clinically significant in $30 \%$ of cases, FES in subclinical form is believed to be occurring in a large percentage of patients with long bone and pelvic fractures, secondary to a rise in intramedullary pressure and venous sinusoidal disruption. Debris of bone and fat gain access to the systemic and pulmonary circulation cause mechanical obstruction as well as multi-organ dysfunction syndrome. FES can be recognised by classic Bergman's triad ${ }^{1}$ characterised by hypoxaemia, petechial rash and mental changes. We present a case of fat embolism syndrome that was reported in a tertiary care centre during the peak of the Covid19 pandemic.

\section{PRESENTATION OF CASE}

In the prevailing pandemic period, the clinical presentation of fat embolism syndrome in a trauma victim may be found to resemble that of Covid-19 pneumonia and ARDS, which may create a dilemma for diagnostic purpose, the implication of this being inadvertent delay in implementation of therapeutic measures. The following account illustrates the case of a thirty-five-year-old with traumatic unilateral fracture femur, admitted during the period of the peak in Covid-19 cases locally, with an inconclusive nasal swab report owing to low sensitivity test, leading to inevitable delay in surgical intervention. Preoperatively this patient developed respiratory failure, which progressed to alteration in sensorium and incidental detection of petechial rash, thus satisfying the Bergman's triad of fat embolism syndrome. After a confirmatory negative swab for SARS-CoV-2, the diagnosis was further ascertained by the detection of retinal fat emboli on fundoscopic evaluation and supported by the fulfilment of Gurd and Wilson, and Schonfeld's criteria. Owing to the pandemic, the provision of high flow nasal oxygen devices in a resource-poor setting like ours facilitated its application in the above case to tide over respiratory failure, enabling successful supportive management in ICU. The patient was operated on at a later date and discharged with an uneventful further course.
Corresponding Author: Dr. Nimisha Mahesh Parkar, 503-B, Estrela by Alcon, Tonca Miramar, Panaji - 403001, Goa, India.

E-mail: nimishaparker@gmail.com

DOI: $10.14260 /$ jemds/2022/59

How to Cite This Article:

Parkar NM, Gomes F, Dessai PP, et al. Fat embolism syndrome - diagnostic dilemma during Covid-19 pandemic. J Evolution Med Dent Sci 2022;11(01):311-314, DOI: 10.14260/jemds/2022/59

Submission 20-12-2021,

Peer Review 14-04-2021,

Acceptance 20-01-2022,

Published 31-01-2022.

Copyright (c) 2022 Nimisha Mahesh Parkar et al. This is an open access article distributed under Creative Commons Attribution License [Attribution 4.0 International (CC BY 4.0)] 


\section{Case Report}

Thirty-five year old male with no co-morbidities presented at an emergency department with trauma to the right thigh, which on X-ray was indicative of a closed intertrochanteric fracture of the right femur. This patient who was apparently alright and haemodynamically stable at admission, with no other injuries, was posted for an orthopaedic procedure after obtaining a Covid-19 swab report as a part of the routine institutional protocol implemented during the pandemic period. Nasopharyngeal swab for rapid antigen test was found to be positive for COVID-19 despite the patient being asymptomatic; hence a repeat oropharyngeal swab was sent for a confirmatory RT-PCR test, as decided by the orthopaedician, which was reported as negative after 24 hours. There was an unfortunate but inevitable delay between the time of trauma and in proceeding with the plan of surgery due to inconclusive Covid-19 status of the patient and prevailing local surge in Covid-19 cases favoured deferral of operative intervention. However a night before the proposed surgery, the patient complained of sudden onset of breathlessness at rest, tachypnoea of 45 breaths per minute, with no orthopnoea or chest pain, no associated cough or fever, oxygen saturation on pulse oximeter dropped to $48 \%$ on room air, improved only to $66 \%$ on supplemental oxygen with simple face mask at 5-8 L/ min. The patient had tachycardia, normal blood pressure, signs of respiratory distress with air entry reduced in bilateral lungs on auscultation and presence of course crepitations in posterior lung fields. Arterial blood gas, on $8 \mathrm{~L} / \mathrm{min}$ of oxygen, revealed $\mathrm{pH}$ 7.46, $\mathrm{pO}_{2} 59.1 \mathrm{mmHg}, \mathrm{pCO}_{2} 30 \mathrm{mmHg}$, Sat $\mathrm{O}_{2}: 66 \%$, $\mathrm{HCO}_{3} 22.4 \mathrm{mmol} / \mathrm{L}, \mathrm{PaO}_{2} / \mathrm{FiO}_{2}$ 118.2. Urgent bedside Chest Xray was suggestive of bilateral diffuse fluffy shadows and was inconclusive.

As a part of preliminary management, upon shifting to the critical care unit, the patient was reassured, placed in the propped up position, and provided supplemental oxygen via high flow nasal oxygen (HFNO) cannula, with incremental flows of 30-50 l/min and FIO2 of 0.5-0.6. ECG was recorded indicating sinus tachycardia with $\mathrm{T}$ wave inversions in lead III. Bedside 2D echocardiogram revealed mild tricuspid regurgitation, mild pulmonary arterial hypertension (Pulmonary arterial systolic pressure of $43 \mathrm{mmHg}$ ) with normal chamber size and biventricular function besides a normal D-dimer value, with no evidence of regional wall motion abnormalities. An urgent CT pulmonary angiography was performed, ruling out the presence of acute pulmonary thromboembolism. However, areas of ground-glass densities were noted scattered in the bilateral lung parenchyma in a centre as well as peripheral distribution, interspersed with areas of interlobular septal thickening, with a minimal bilateral pleural effusion. Although these features were primarily suggestive of ARDS, a prior inconsistency in Covid19 status compelled the physicians to order a third repeat swab for RT- PCR, which was reported to be negative. The patient was immediately started on Low Molecular Weight Heparin ( Inj enoxaparin $60 \mathrm{mg} \mathrm{s} / \mathrm{c}$ twice daily), along with oral high dose atorvastatin and intravenous methylprednisolone based on suspicion of fat embolism.

Subsequently, the patient began to show behavioural abnormalities which evolved from anxiety and apprehension (Richmond Agitation and Sedation Score: RASS +1) to being aggressive and attempting to pull intravenous lines and catheters (RASS +3 ) within 8-12 hours, hence compromising compliance to HFNO supplementation. No abnormalities were noted in computed tomography of the brain. This warranted administration of sedation of inj midazolam $1 \mathrm{mg}$ IV bolus followed by infusion of $1 \mathrm{mg} /$ hour, with meticulous monitoring for signs of respiratory depression and titrating sedation to achieve RASS score up to -1 (awakens to voice with eye contact for more than 10 seconds).

Laboratory investigations revealed a significant drop in haemoglobin, platelets and haematocrit as compared to the baseline values and admission, no evidence of bleeding and with normal renal and liver biochemistry. Optimal cooperation from the patient for sustained delivery of HFNO, ensured favourable improvement in oxygenation status and ventilation as reflected in serial arterial blood gas reports.

A strong suspicion of fat embolism based on classical history and presentation was made. Further clarity to this differential diagnosis was provided by a fundoscopic evaluation which vividly revealed the presence of a distinct fat embolus in the inferotemporal quadrant of the right eye, described as Purtscher retinopathy. However, a routine urine examination did not reveal the presence of fat globules. Retrospective head to toe examination revealed the presence of indistinct petechial rash over the back of the neck and bilateral axillae.

A positive radiological resolution and clearance of infiltrates by end of one week encouraged weaning from HFNO and the patient was eventually discharged from the intensive care unit to the orthopaedics ward, where he underwent a planned surgical fixation of fracture under regional anaesthesia, with an uneventful course until discharge from hospital.

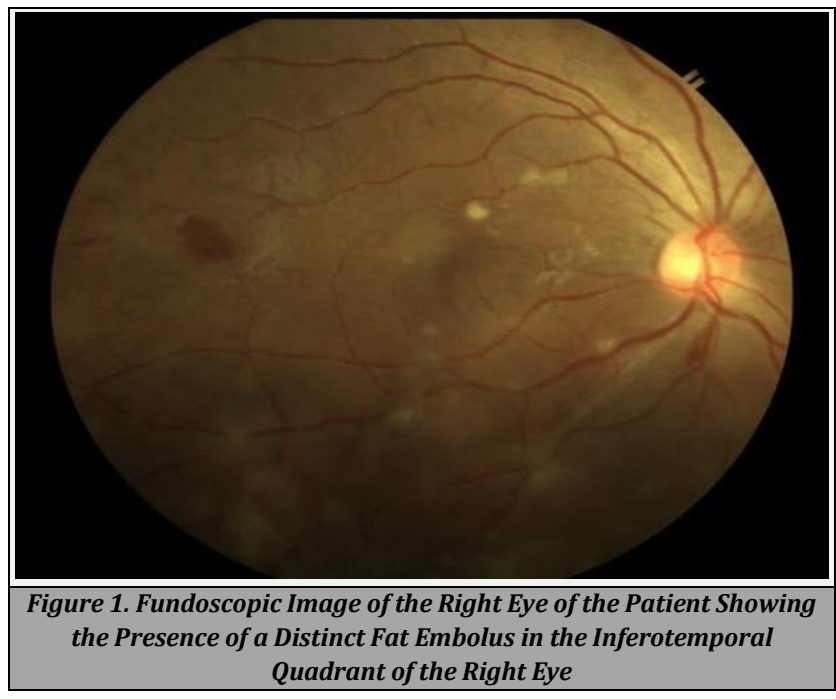




\begin{tabular}{|c|c|c|c|c|c|}
\hline \multirow{4}{*}{ FES Diagnosis } & \multicolumn{4}{|c|}{ Clinical Criteria for diagnosis of Fat Embolism Syndrome and their apllication in the above case } & \\
\hline & \multicolumn{2}{|c|}{ Gurd and Wilson's Criteria } & \multicolumn{3}{|c|}{ Schonfeld's Criteria } \\
\hline & \multicolumn{2}{|c|}{2 Major or 1 Major +4 Minor } & \multicolumn{3}{|c|}{ Cumulative score of $>5$ is needed } \\
\hline & & & & Score & \\
\hline \multirow[t]{4}{*}{ Major } & Petichae & $\checkmark$ & Petichae & 5 & $\checkmark$ \\
\hline & Hypoxemia & $\checkmark$ & $\mathrm{X}$-ray infiltrates on chest & 4 & $\checkmark$ \\
\hline & Altered Mentality & $\checkmark$ & Hypoxaemia & 3 & $\checkmark$ \\
\hline & & & Mental confusion & 1 & $\checkmark$ \\
\hline \multirow[t]{9}{*}{ Minor } & Tachycardia & $\checkmark$ & Tachycardia & 1 & $\checkmark$ \\
\hline & Fever & & Fever & 1 & \\
\hline & Thrombocytopenia & $\checkmark$ & Tachypnea & 1 & $\checkmark$ \\
\hline & Unexplained anaemia & $\checkmark$ & & & \\
\hline & Anuria or oliguria & & & & \\
\hline & Retinal embolism & $\checkmark$ & & & \\
\hline & $\begin{array}{l}\text { Fat globules in urine or } \\
\text { sputum }\end{array}$ & & & & \\
\hline & Jaundice & & & & \\
\hline & High ESR & & & & \\
\hline $\begin{array}{l}3 \text { major }+4 \text { minor } \\
\text { present in above case }\end{array}$ & & & & Total score & 15 \\
\hline
\end{tabular}

\section{DISCUSSION}

Fat embolism syndrome is a clinical entity, the description of which was first given by Zenker, over 150 years ago, 2 trauma being its leading aetiology. Young age, closed fractures, multiple fractures, and conservative therapy for long-bone fractures have been cited to be eminent risk factors for the development of FES. ${ }^{3}$

The prevalence of the SARS-CoV pandemic broadens the differential diagnosis in clinical scenarios as encountered in the above case. Studies have shown significantly lower sensitivity and specificity of rapid antigen test kits as compared to real-time PCR test, ${ }^{4}$ which dictated repetition of Covid-19 swab and favoured fat embolism syndrome as a diagnosis of exclusion. A high index of suspicion facilitated the diagnosis of fat embolism syndrome as its incidence is only about 1-11 \% in trauma victims with long bone fractures.5,6 Diagnosis was further ascertained by Gurd and Wilson criteria ${ }^{7}$ as well as Schonfeld's criteria ${ }^{8}$ which verified our clinical scepticism. Studies showed that the presence of fat globules either in urine or sputum is not necessary for confirming the diagnosis and its presence is said to be of uncertain significance.

The enhanced utility of HFNO even in resource-poor institutions during the COVID pandemic has highlighted its application in tiding over the crisis and preventing the need for invasive ventilation in FES associated type 1 respiratory failure. ${ }^{9}$ Along with the resolution of dyspnoea and improved oxygenation, it is an appealing alternative to non-invasive ventilation with cumbersome oronasal mask interfaces, HFNO provides enhanced cooperation among patients with FES having neurological manifestations, minimal dead space, effecting positive pressure ventilation and ensured FIO2, along with the advantage of heated humidified air which enables mucociliary clearance.

Based on current evidence it has been suggested that steroids, particularly methyl prednisolone may be of benefit in preventing FES and the resultant hypoxia when started in individuals with traumatic long bone fractures with no demonstrable mortality benefits as a therapeutic modality. ${ }^{10}$ Low dose steroid was administered in our patient with no evident development of postoperative infection. High dose statins have been tried in FES in relation to their antiinflammatory effect. ${ }^{11}$

As preventive measures of fat embolism syndrome and its consequences, early fracture fixation within 24 hours of trauma is recommended and is ideal. ${ }^{12}$ There is limited evidence suggesting the use of intravenous albumin for the resuscitations of blood volume following trauma, which enables fatty acid binding and is hence implicated in ameliorating injury to the lungs. ${ }^{13}$

\section{CONCLUSIONS}

Early diagnosis based on clinical suspicion, ruling out differential diagnostic conditions, and punctilious supportive treatment remains the mainstay modality of management in intensive care setup in trauma victims with above-described presentation.

Financial or other competing interests: None.

Disclosure forms provided by the authors are available with the full text of this article at jemds.com. 


\section{REFERENCES}

[1] Rodríguez-Gutiérrez R, Rodarte-Shade M, GonzálezGonzález JG, et al. Bergman's triad: fat embolism syndrome. Am J Med Sci 2015;349(2):186.

[2] Groebler LK, Liu J, Shanu A, et al. Comparing the potential renal protection activity of desferrioxamine $\mathrm{B}$ and novel chelator desferrioxamine B-N-(3hydroxyadamant-1-yl) carboxamide in a cell model of myoglobinuria. Biochem J 2011;435(3):669-77.

[3] Dillerud E. Abdominoplasty combined with suction lipoplasty: a study of complications, revisions, and risk factors in 487 cases. Ann Plast Surg 1990;25(5):333-8.

[4] Kruttgen A, Conelissen CG, Dreher M, et al. Comparison of the SARS CoV-2 Rapid Antigen test to the Real Star Sars -CoV-2 RT PCR Kit. J Virol Methods 2020;288:114024.

[5] Pell AC, Christie J, Keating JF, et al. The detection of fat embolism by transoesophageal echocardiography during reamed intramedullary nailing. A study of 24 patients with femoral and tibial fractures. J Bone Joint Surg $\mathrm{Br}$ 1993;75(6):921-5.

[6] Riska EB, Myllynen P. Fat embolism in patients with multiple injuries. J Trauma 1982;22(11):891-4.

[7] Gurd AR, Wilson RI. The fat embolism syndrome. J Bone Joint Surg Br 1974;56B(3):408-16.

[8] Dhar D. Fat embolism syndrome. J Coll Phys Surg Pak 2012;22(12):800-2.

[9] Ferrao JB, Amaral M, Ilharco M, et al. High-flow nasal cannula oxygen therapy in pulmonary fat embolism-case report. Austin Crit Care Case Rep 2021;5(1):1019.

[10] Bederman A, Daniaux M, Felber S, et al. MRI findings in cerebral fat embolism. Eur Radiol 1998;8(9):1590-3.

[11] Whalen LD, Khot SP, Standage SW. High-dose rosuvastatin treatment for multifocal stroke in traumainduced cerebral fat embolism syndrome: a case report. Pediatr Neurol 2014;51(3):410-3.

[12] George J, George R, Dixit R, et al. Fat embolism syndrome. Lung India 2013;30(1):47-53.

[13] Abbot MG. Fat embolism syndrome: an in-depth review. Asian Journal of Critical Care 2005;1:19-24. 